\title{
Antimicrobial and Antioxidant Properties of Common Indian Medicinal Plants
}

\author{
Vishakha, Pratibha Teotia and Prerna* \\ Department of Biotechnology (SET), Noida International University, Greater Noida, India \\ Corresponding author email: prerna@niu.edu.in
}

\section{ABSTRACT}

The Vedas point out Indian medicinal herbs as having healing properties. Since time immemorial, the humans of this vicinity have utilized them to deal with endemic illnesses along with malaria, in addition to pores and skin illnesses, not unusual place pains, and fevers, and they're referred to as to as "jadibuti." The development of recent lines of contamination-inflicting difficulty depends specialists, in addition to the flip of activities and unfold of antimicrobial check, are of superb situation to the general prosperity neighborhood. Commonly used remedial vegetation of our community be an incredible wellspring of drugs to combat down this issue. The antibacterial effect of a few selected Indian restorative vegetation became assessed on a few bacterial lines. Therapeutic vegetation has an inborn capacity to limitation pathogenic natural entities. This assignment makes a specialty of 5 plant species which might be not unusual place withinside the Eastern vicinity of the world, mainly the Indian subcontinent. Although large research has been executed through manner of way of several scientists, now not a bargain is thought about the antimicrobial and antioxidant houses of moringa, gotu kola, adusa, agathi and golden shower. Hence, this test has a bent to evaluate the identical for the cause of future reference withinside the region of producing broad-spectrum medicinal drugs from vegetation. Agar well-diffusion method, Total Phenolic Content, Total Flavonoid Content, Ferric Reducing Antioxidant Power, and DPPH have been used to check leaf extracts of the 5 vegetation in ethanol for his or her hobby towards 3 bacterial species and antioxidant hobby at $200 \mathrm{mg} / \mathrm{ml}$ concentrations.

\section{KEY WORDS: ANTIMICROBIAL, ANTIOXIDANT, AGAR-WELL DIFFUSION METHOD, DPPH AND FRAP.}

\section{INTRODUCTION}

During the numerous apparently all the time there was a developing sales withinside the exam of widespread flora and their remedial really well worth in one-of-akind bits of the world. The remedial houses of flora were investigated in view in their strong pharmacological activities, low harmfulness and financial practicality. This rebuilding of hobby in plant derived medicines is prevalently a end result of the contemporary unpreventable conviction that inexperienced prescription is secured and greater reliable than the acute fabricated drug, an intensive a part of which can also additionally have opposed outcomes. (Chew, 2012).

Biosc Biotech Res Comm P-ISSN: 0974-6455 E-ISSN: 2321-4007
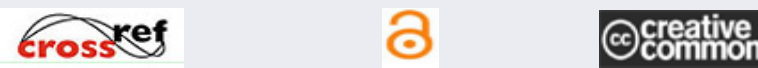

Identifiers and Pagination

Article Information

Year: 2021 Vol: 14 No (9) Special Issue

Pages: $121-128$

Received: 04 $4^{\text {th }}$ May 2021

This is an open access article under Creative

Commons License Attribn 4.0 Intl (CC-BY).

DOI: $h t t p: / / d x . d o i . o r g / 10.21786 / b b r c / 14.9 .25$
Standard scientific remedy makes use of flowers and those can also additionally helper metabolites and fundamental oils that may be used to deal with illnesses. It is on this way vital to research the antimicrobial homes of those ethnomedicinal flowers which might be constantly now no longer pharmacologically idea of and clinically attempted. In the Philippines, its plant networks are special a lot that limitless concentrates from community useful flowers may be used as professionals in opposition to microbial microorganisms. Around two decades earlier, most effective a chunk of a part of this plant collection had long gone via drug screening. (Selvamohan T, 2012).

Restorative plants have been referred to for centuries and are considered as a rich wellspring of drug specialists for the counteraction and treatment of sicknesses and afflictions. As indicated by WHO, over $80 \%$ of the populace inside agricultural nations utilizes natural and other customary medications to treat their regular afflictions. (WHO,1998) Nature has bestowed Oman with an sizable wealth of medicinal flora which can be 
extensively utilized in conventional machine of medicine. (Ghazanfar SA,1993) Moringa oleifera normally called pony radish or drumstick tree in English, has an area with own circle of relatives Moringaceae. It is a touch predicted tree, that's nearby to South Asia, Africa and Arabia and is applied as traditional remedy in several tropical and subtropical nations.

It is a deciduous tree growing quick even in helpless soils, all round adjusted to dry seasons and geared up to attain up to fifteen $\mathrm{m}$ in tallness. It is one of the 14 varieties of kind Moringa, that's nearby to India, Africa, Arabia, Southeast Asia, the Pacific and Caribbean islands, and South America. The blossoms and the natural merchandise display up double each year, and seeds or cuttings are applied to engender the tree. Practically each one of the portions of M. oleifera are applied for special ailments withinside the local remedy of South Asia,counting the remedy of diabetes, hypertension, infection and impossible to resist ailments. Its leaves, gadgets and blossoms are for the maximum component gobbled for sustenance. Alhakmani, F, (2013).

Flavonoids are a gathering of polyphenolic compounds with known properties which incorporate free extremist rummaging, hindrance of hydrolytic oxidative catalysts and calming activity. In the current investigation, the relative lipid peroxidation restraint movement was done with chosen therapeutic plants; Golden Shower (Cassia fistula), Adusa (Adhatoda vasica), Agathi (Sesbania grandiflora), Gotu Kola (Centella asiatica), and (Moringa oleifera). (P. Suresh Kumarl,2008) Moringa oleifera Lam. (M. oleifera), commonly known as horseradish or drumstick tree in English, belongs to family Moringaceae. It is a small sized tree, which is native to South Asia, Africa and Arabia and is used as traditional medicine in many tropical and subtropical countries. (Moyo B,2011).

Cassia fistula having a place with Leguminosae family has been chosen. In the Indian writing, this plant has been depicted to be valuable against skin sicknesses, liver inconveniences, tuberculosis organs and its utilization into the therapy of hematemesis, pruritus, leukoderma, and diabetes has been recommended. (Alam MM,1990, Asolkar LV,1992) Cassia fistula plant organs are known to be a significant wellspring of auxiliary metabolites, Indian individuals are utilizing the leaves to treat aggravation; Cassia fistula plant organs are known to be a significant wellspring of optional metabolites, prominently phenolic compounds. (Morimoto S,1988) Adhatoda vasica and Sesbania grandiflora Pers are the two significant restorative plants local to India and were utilized in Indian medication since old occasions. a. It is utilized as expectorant, antispasmodic, bronchodilator, hostile to histaminic, uterine energizer, utilized in the treatment of feminine issues, eye contaminations, skin infections, sore throat, draining loose bowels and has narcotic properties.

(U.P. Claeson,2000) Sesbania grandiflora Pers (own circle of relatives Fabaceae) an decorative tree, is a peoples treatment for bruises, catarrh, dysentery, fevers, headaches, rheumatism, small pox and stomatitis. According to Ayurveda culmination are used for the remedy of anemia, bronchitis and tumors. (Kirtikar,1995) Centella asiatica Urban (Umbelliferae/Apiaceae family) is commonly known as Mandukparni. It is an enduring crawling spice, widely advanced as a vegetable or zest in India. Since historical times, it's been used as a reminiscence enhancing, electricity promoting, wound healing, immune booster, anti-anxiety, ant epilepsy and anti-strain substance. (Kartni T.1998, Chen Y,2003) Centella asiatica has been clinically applied in intellectually hindered kids and moreover in remedy of anxiety intellectual issues. This plant is also determined to enhance short-term reminiscence and learning. Centella asiatica has moreover proven a shielding effect in opposition to oxidative damage added approximately through lead acetic acid derivation actuated neurotoxicity. (Meena,2012).

Review of Literature: Sometime before humanity found the presence of microorganisms, the possibility that specific plants had recuperating potential, surely, that they contained what we would presently portray as antimicrobial and cancer prevention agent standards, was well accepted. Since vestige, man has utilized plants to treat normal irresistible infections and a portion of these conventional drugs are as yet included as a component of the ongoing treatment of different ailments. (Calixto,2005).

Countless therapeutic plants have been perceived as important assets of common antimicrobial mixtures as an elective that can possibly be compelling in the treatment of these hazardous bacterial contaminations. According to the World Health Organization (WHO), medicinal plants would be the best source to obtain a variety of drugs. Sarita (Manandhar,2019) In recent years, secondary plant metabolites (phytochemicals), previously with unknown pharmacological activities, have been extensively investigated as a source of medicinal agents. (Krishnaraju AV,2005) Thus, it is anticipated that phytochemicals with adequate antibacterial efficacy will be used for the treatment of bacterial infections. (Balandrin MF,1985).

\section{Figure 1: Moringa (Cassia fistula)}

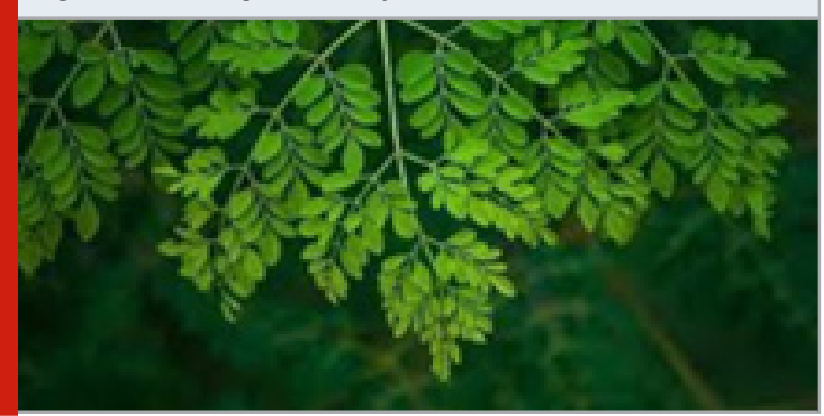

MATERIAL AND METHODS

Materials: The plants used in this study are in popular 
demand throughout the nation, and I easily obtained them from the local community gardens in my neighborhood. This name is likewise utilized for Cassia fistula, the brilliant downpour tree. Moringa is filled in home nurseries and as living wall in South Asia and Southeast Asia, where it is normally sold in nearby business sectors. The bark, sap, roots, leaves, seeds and blossoms are utilized in customary medication. Exploration has analyzed what it may mean for blood lipid profiles and insulin emission.

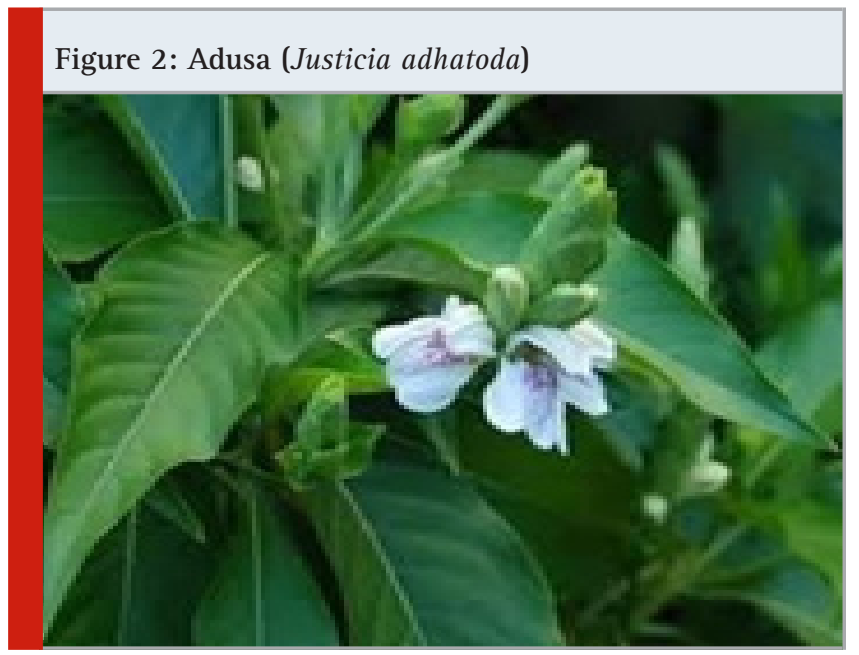

Figure 3: Agathi (Sesbania grandiflora)

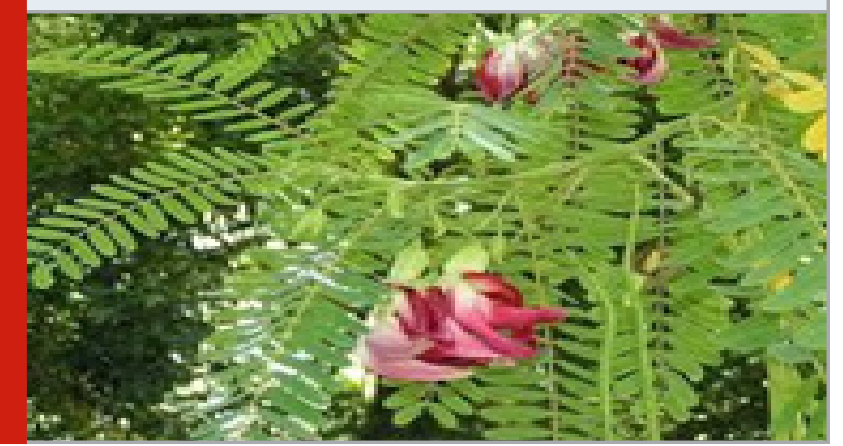

Figure 4: Gotu Kola (Centella asiatica)

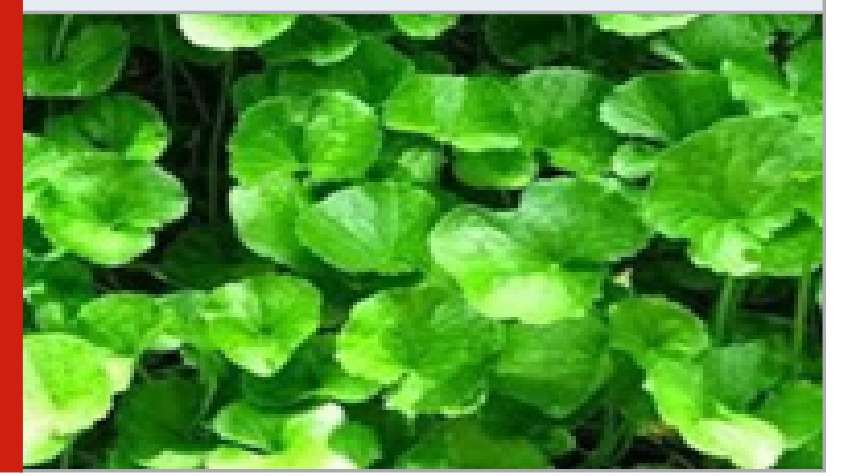

Adusa: Justicia adhatoda, commonly known in English as Malabar nut, adulsa, adhatoda, vasa, vasaka. Leaves of justicia adhatoda contains phytochemicals such as alkaloids, tannins, saponins, phenolics and flavonoids. This shrub has a number of traditional medicinal uses in siddha medicine, Ayurvedic, Homeopathy and Unani system of medicine.

Agathi: Sesbania grandiflora, generally known as vegetable hummingbird, West Indian pea, agati, or katurai, is a little tree in the class Sesbania in the vegetable family. It has consumable blossoms and leaves generally eaten in the Southeast Asia and South Asia. The leaf concentrate may repress the development of cutting-edge glycation finished results.

Figure 5: Golden Shower (Cassia fistula)

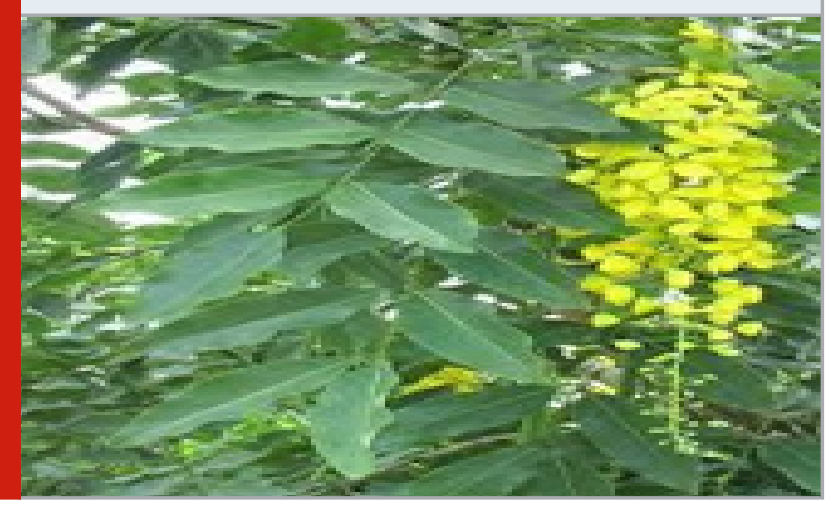

Figure 6: Aeromonas hydrophila

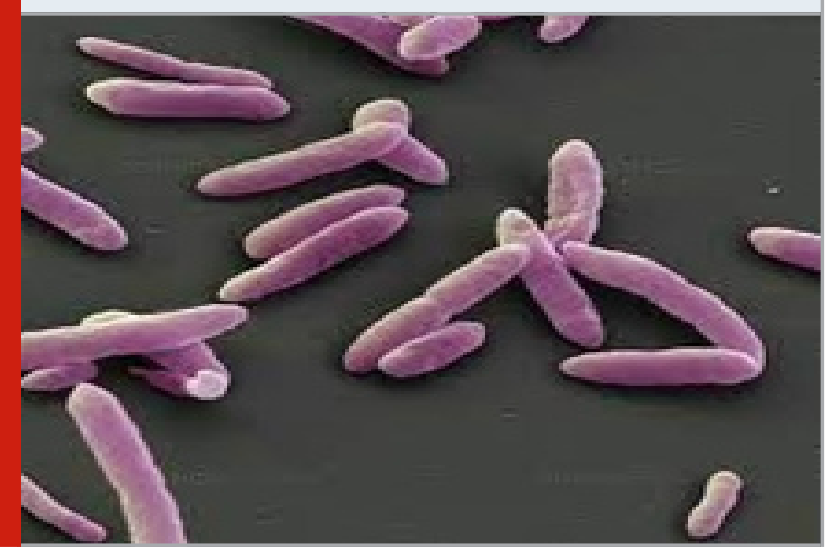

Figure 7: Pseudomonos

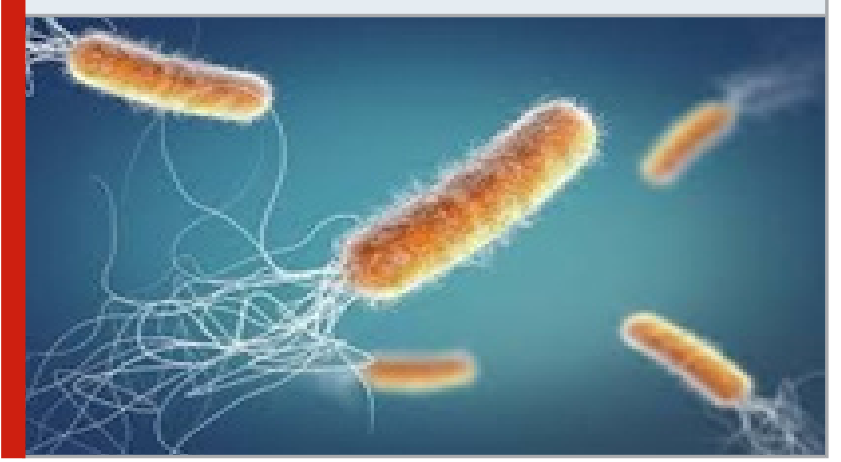

Centella asiatica, normally known as Gotu Kola, brahmi, Indian pennywort and Asiatic pennywort, is a herbaceous, lasting plant in the blooming plant family Apiaceae. In customary medication, Centella asiatica has 
been utilized to treat different issues and minor injuries, albeit clinical adequacy and security have not been deductively affirmed. Brilliant shower: Cassia fistula, generally known as brilliant shower, cleansing cassia, Indian laburnum, or pudding-pipe tree, is a blossoming plant. It is the state blossom of Kerala in India.

Human pathogenic bacterial species: Pathogenic strains of gram-positive bacteria Aeromonas hydrophila, as well as gram-negative bacteria Pseudomonas and Escherichia coli were obtained from Helix Biogenesis Laboratory, Noida and stored at 4 degrees $C$ as nutrient broth cultures for further assay for susceptibility towards the medicinal plant extracts on MHA media.

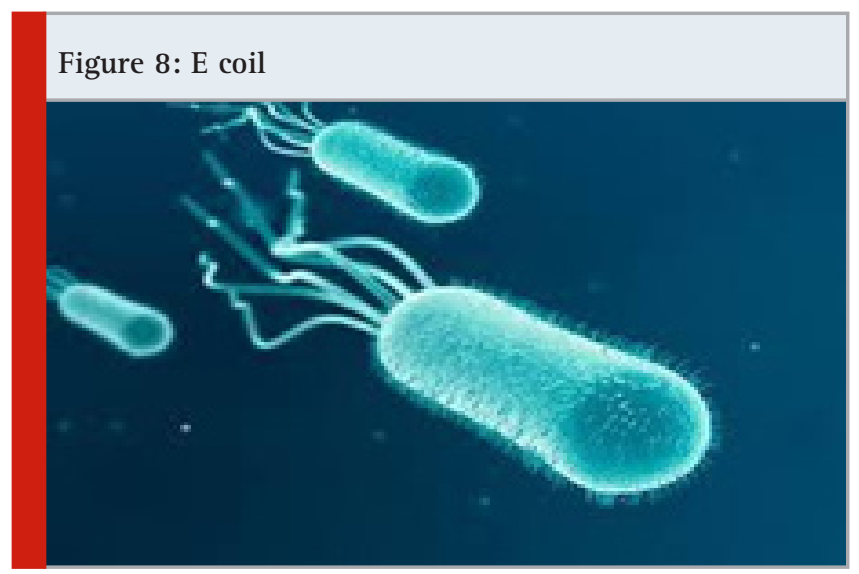

Figure 9: Dried leave powder

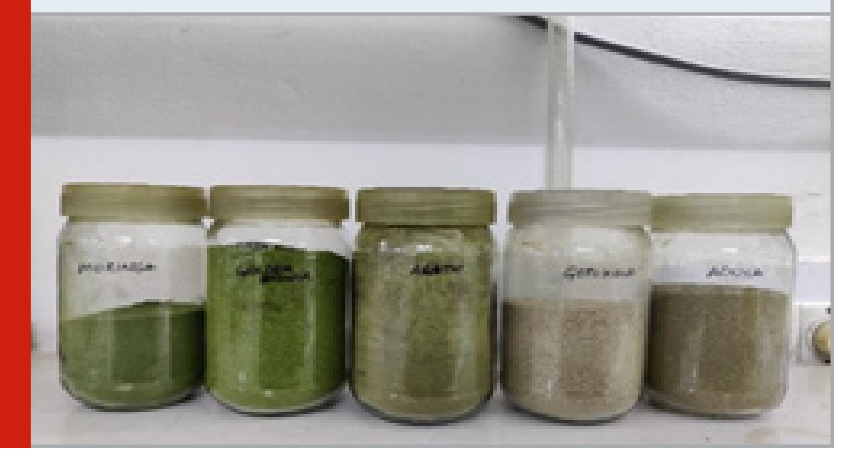

Figure 10: $10 \mathrm{gm}$ plant extract dissolved in $10 \mathrm{ml}$ of ethanol

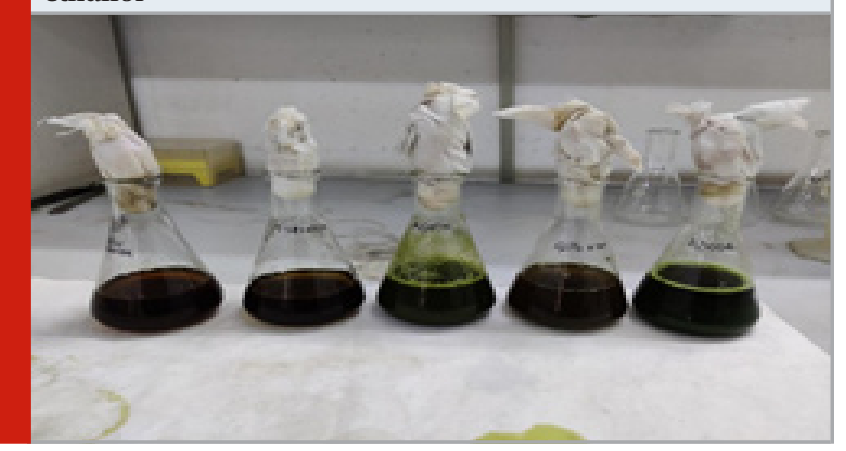

Aeromonas hydrophila is a heterotrophic, Gram-negative, bar formed bacterium chiefly found in regions with a warm environment. This bacterium can be found in new or bitter water. It can make due in vigorous and anaerobic climate and can process materials like gelatin and hemoglobin. Pseudomonas is a typical sort of microbes, which can make diseases in the body in specific situations. There are a wide range of kinds of Pseudomonas microscopic organisms. A couple of types can cause a disease. Pseudomonas microscopic organisms tend to live and raise in water, soil, and sodden regions.

Escherichia coli is a kind of microbes that ordinarily lives in your digestive organs. It's likewise found in the gut of certain creatures. Most sorts of E. coli are innocuous and even assistance keep your stomach related plot sound. However, a few strains can cause looseness of the bowels in the event that you eat sullied food or drink fouled water.

\section{Preparation of plant extracts}

1. Separate the leaves of each plant from its stems (or any other plants parts). Leaves were thoroughly washed with distilled water to remove any traces of dust and dirt present on the plants. Plants were then dried under complete shade.

2. Next, leaves were grounded in a standard kitchen grinder and stored in separate sterile glass bottles.

3. Next, I weighed out $10 \mathrm{~g}$ of each plant leaves and incubated them in $50 \mathrm{ml}$ of ethanol at room temperature for a period of 24 hours.

4. Plants are filtered using funnel and standard laboratory filter/blotting paper.

5. The resulting liquid obtained is kept to dry in sterile petri plates. Methanol vaporizes overnight. However, plants with water solvent require drying in hot air oven.

6. When plates are completely dried, scrap off the leftover plants on the surface of the plates with a scalpel or blade, and store in fresh Eppendorf tubes at 4 degrees $\mathrm{C}$ temperature.

7. The final extracts so obtained are ready to be further assessed for their antibacterial properties by agar well-diffusion method and antioxidant properties by total phenolic content, total flavonoid content, DPPH and FRAP.

\section{Methods}

1. Screening for antimicrobial activity by agar-well diffusion method

Mueller-Hinton Agar (MHA) is the standard media for antimicrobial susceptibility testing (AST) of various clinical isolates (here, medicinal plant extracts). The WHO has accepted and recommended this media for extensive AST due to its following properties:

- It demonstrates good batch-to-batch reproducibility for susceptibility testing.

- It is low in tetracycline and sulfonamide inhibitors.

- It supports the growth of the most non-fastidious bacterial pathogens 
MHA agar was prepared by standard protocol adopted from (Jan Hudzicki,2009), and sterilized by autoclaving at 15 lbs pressure (121 degrees C) for 15 minutes. This was then poured into sterile petri plates (approx. $35 \mathrm{ml}$ per plate), and allowed to solidify. 200 microlitre cultures of each of the 3 bacterial species was poured using a pipette onto separate sterile MHA plates, ethanolic extracts of the plants under study, and uniformly distributed throughout the surface areas of the plates by generalized spreading technique. Wells of diameter 6- $8 \mathrm{~mm}$ were then punched onto each plate for the 5 plant extracts in use, ethanol extracts of the plants. 150microliter of each plant extract was poured into the wells corresponding to their name on the respective plates, those of., Aeromonas hydrophila, Escherichia coli, as well as Pseudomonas. These were then incubated at 37 degrees $C$ for a period of 24 hours and observed for their zone of inhibition*against the respective bacterial strains, thereafter.

\section{Estimation of phenolic content Aim: Total Phenolic Content}

Principle: Phenolic evaluation measure depends on folin ciocalteu technique. The strategy depends on the exchange of electrons in antacid medium from phenolic compound to frame a blue chromophore established by phosphotungstic complex where the greatest assimilation relies upon the centralization of phenolic compound. In the first F.C. test, carbonate cushion is utilized from $\mathrm{pH}$ change. Quercetin is utilized as the reference standard compound. ( Singleton,1999).

Material required: Gallic acid, 20\% NaNO3, 0.5N FCR and distilled waster

\section{Procedure}

1. $1 \mathrm{ml}$ sample extract added to the tube.

2. Quercetin as standard was added.

3. $100 \mu l$ FCR was added.

4. Incubate for 10 mins at for temperature

5. 2,5 ml NaNO3 was added

6. Incubate at room temperature for 30 mins.

7. Absorbance measured at 760nm.

\section{Estimation of flavonoid content}

Aim: Total flavonoid content

Principle: Determination by aluminum chloride color metric method. Aluminum chloride forms complex with the hydroxide group of flavonoids present in sample. Formation of acid stable complexes with C-4 keto group and either $\mathrm{C}-5$ hydroxide group off flavones and flavanols in addition with aluminum chloride. (Jiao,2000) Aluminum chloride also forms acid labile complexes with ortho di-hydroxide group in the A or B ring of flavonoids for building the calibration curve quercetin is used as a standard material. This complex has the maximum observance at $420 \mathrm{~nm}$.

Material required: $10 \% \mathrm{AlCl} 3$, methanol, quercetin, $1 \mathrm{M}$ potassium acetate distilled water.

\section{Procedure}

1. Prepare $1 \mathrm{mg} / \mathrm{ml}$ quercetin stock solution and prepare different concentration from $10 \mu \mathrm{g} / \mathrm{ml}$ to $200 \mu \mathrm{g} / \mathrm{ml}$

2. Blank consist of all reagent except quercetin.

3. $1 \mathrm{ml}$ from each sample.

4. $3 \mathrm{ml}$ methanol was added

5. $200 \mu \mathrm{l}$ ALCL3 was added.

6. $200 \mu \mathrm{l}$ potassium acetate was added.

7. $3 \mathrm{ml}$ distilled water was added.

8. Incubate at room temp. for 30 mins.

9. Absorbance measured at 420nm.

\section{Estimation of antioxidant activity by DPPH Aim: Determine antioxidant activity.}

Principle: 2,2-Diphenyle-1-picrylhydrazyl (DPPH) is a stable free radical method is an antioxidant assay based on electron-transfer that produces a violet solution in ethanol. Antioxidant react with DPPH and reduce it to DPPH-H and as consequence the absorbance decrease. DPPH then acted upon an antioxidant it converted into diphenyl picryl hydrazine. This can be identified by the conversion of purple to light yellow color. That means the antioxidant effect is proportional to the disappearance of the DPPH in test samples. (Lee, J.,2007)

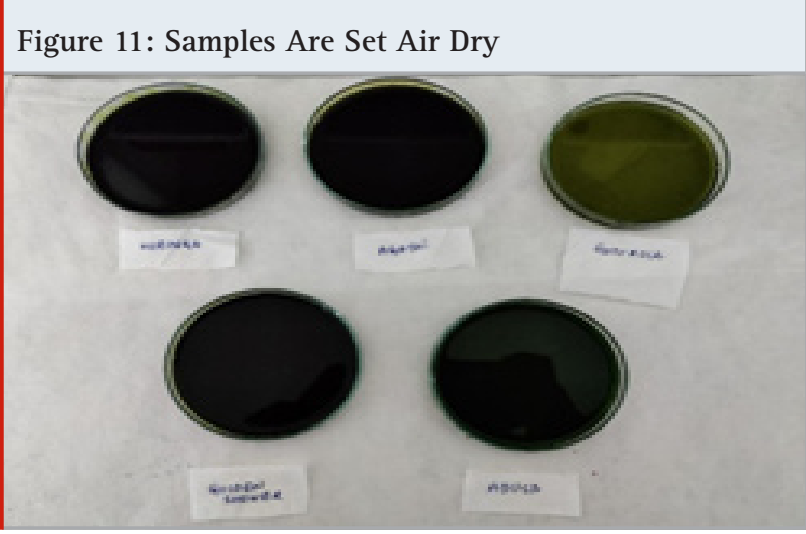

Figure 12: Air drier samples

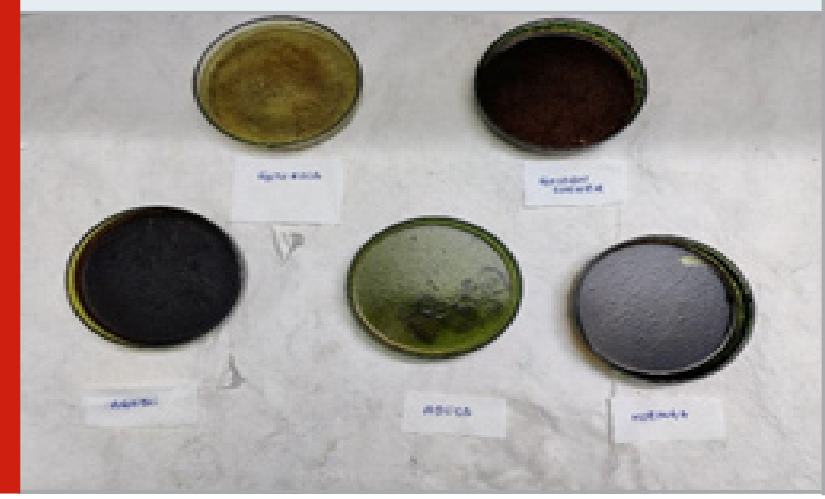

Material required: Ethanol, $0.1 \mathrm{mM}$ DPPH solution (in ethanol), DMSO, distilled water, samples

\section{Procedure}

1. 2-20 $\mathrm{kl}$ of plant extract (final vol. $40 \mu \mathrm{l}$ with 
DMSO)

2. $2.96 \mu \mathrm{l}$ of DPPH solution $(0.1 \mathrm{mM})$

3. Incubate for 20 mins at room temperature.

4. Observed absorbance at 517nm

$\%$ RSA = Abs (control) - abs (sample) / Abs (control) X 100

Control: $3 \mathrm{ml}$ of DPPH solution

5. Estimation of antioxidant activity by FRAP

Aim: Ferric reducing antioxidant power

Figure 13: Prepared ethanolic plant extract

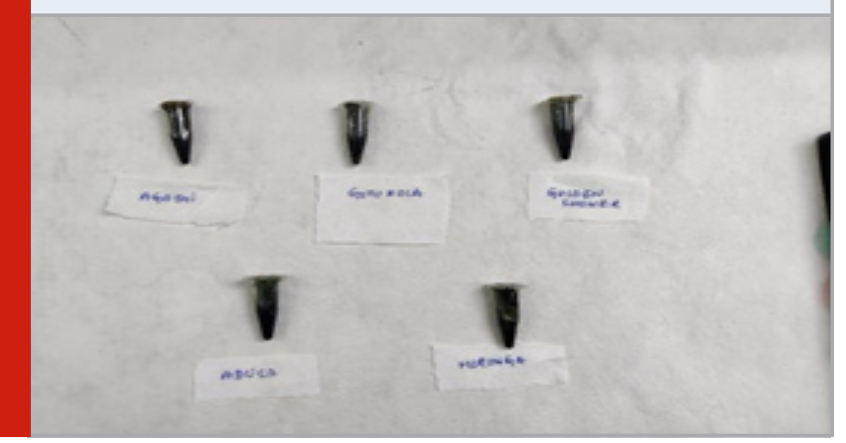

Principle:

Ferric reducing antioxidant power (FRAP) was determined by the method of Benzie \&t strain.

(Benzie, 1996)

Figure 14: Ethanol extract against aeromas hydrophila

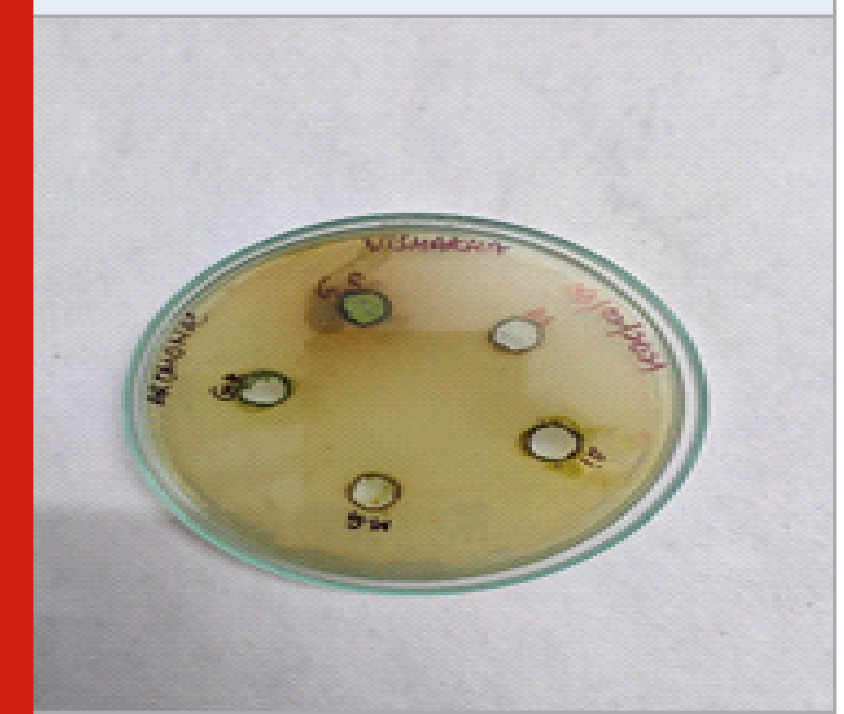

Material required: Acetate buffer (300mM, pH 3.6), 2,4,6-tripyridye-s-traizine (TPTZ) $(10 \mathrm{mM}$ in $40 \mathrm{~mm}$ of $\mathrm{HCl})$, Fecl3.6H20 (20mM)

\section{Procedure}

1. $0.1 \mathrm{mLnof}$ ethanolic extract was taken in test tube.

2. $2 \mathrm{ml}$ pre-warmed FRAP reagent was added $\mathrm{Ct}$ solution was incubated at room temperature for 10 mins.
3. Absorbance was measured at 593nm.

Note - FRAP reagent was prepared freshly \& consisted of acetate buffer, TPTZ and Fecl3.6H2O mixed in ratio 10:1:1 respectively:

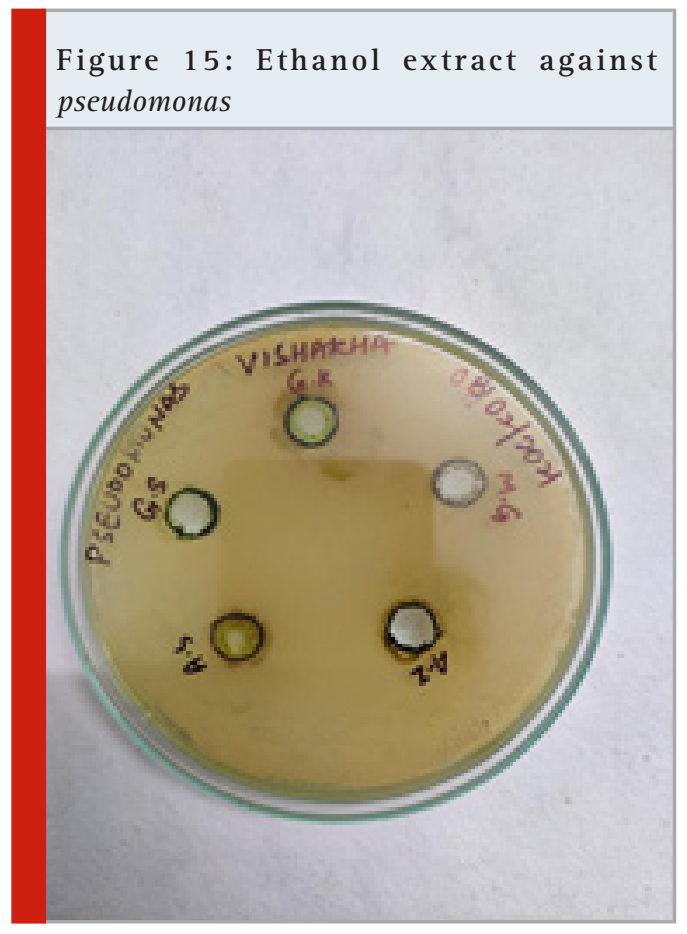

Figure 16: Ethanol extract against E.coli

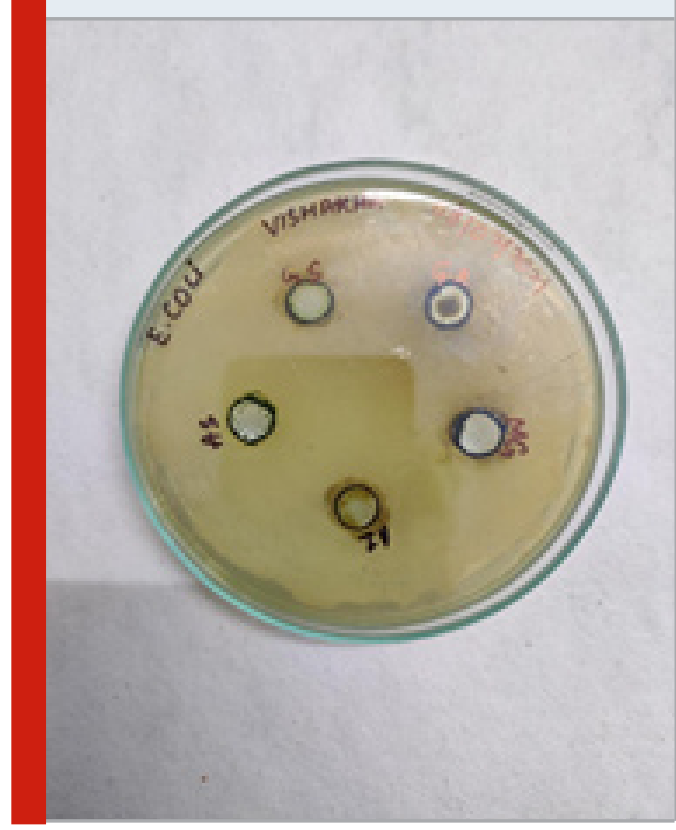

\section{RESULTS}

\section{Antimicrobial activity}

Ethanol extracts of Agathi, Adusa, Golden shower, Moringa and Gotu kola showed no ZOI against Aeromonas hydrophila, Pseudomonas and E.coli. 

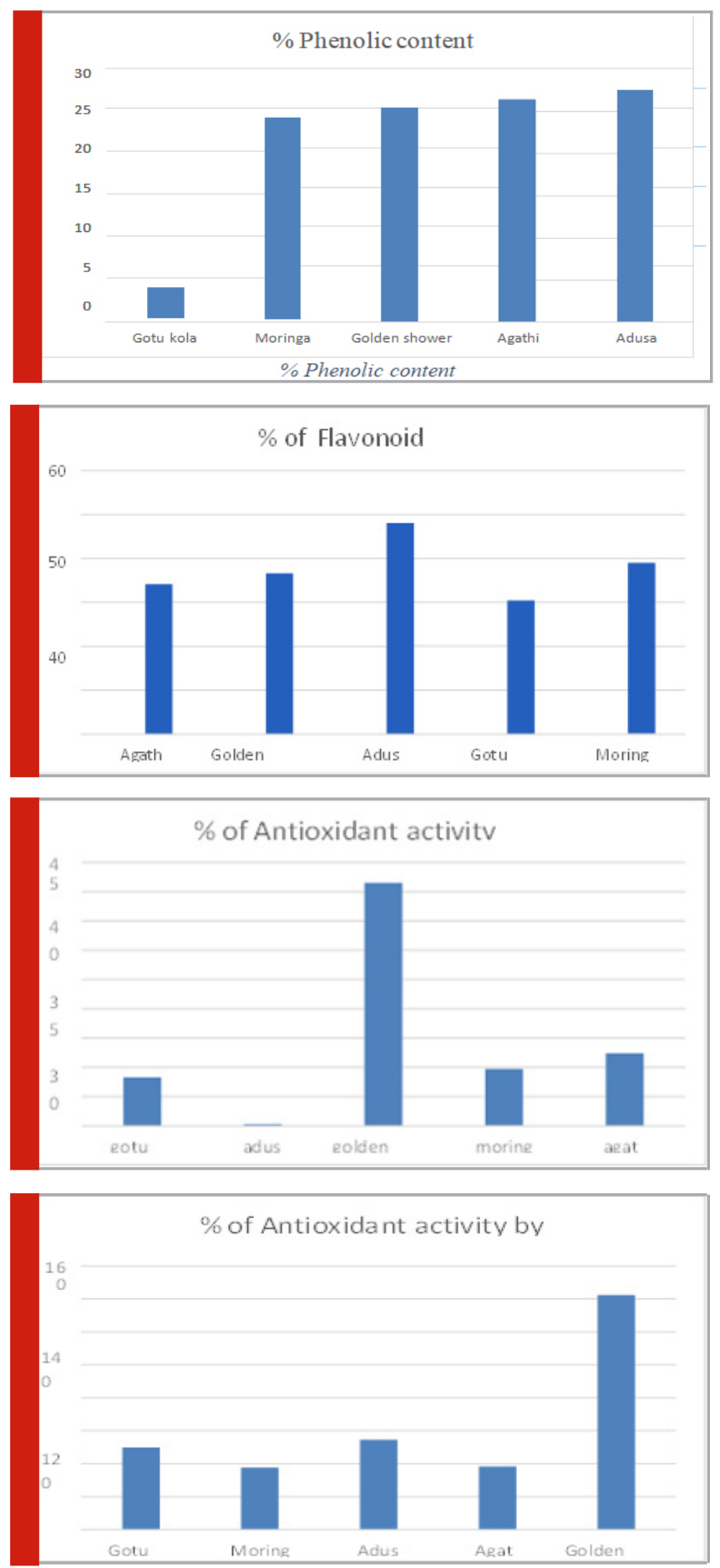

Estimation of phenolic content: The plant samples phenolics are composed of phenolic acids, flavonoids, tannins and proanthocyanidins. Plant samples shows low phenolic content and lowest phenolic content was present in Gotu kola. The phenolic content of the other samples I,e Moringa (23.95468), Golden shower (25.16003), Agathi (26.34363) and Adusa (27.47892) respectively.

Estimation of flavonoid content: Flavonoids are structurally diverse secondary metabolites in plants, with a multitude of functions. These span from functions in regulating plant development, pigmentation, and UV protection, to an array of roles in defense and signaling between plants and microorganisms. Plant sample Adusa has been found to have highest flavonoid content i.e $48.052 \%$. In rest of the samples the flavonoid content lies between $30-38 \%$.

Estimation of antioxidant activity by DPPH: The antioxidant in plant samples are mainly polyphenols (phenolic acids, flavonoids, anthocyanins, lignans and stilbenes), carotenoids (xanthophylls and carotenes) and vitamins. out of five sample, four samples has antioxidant activity in range of $0.255-12.388 \%$ discoloration. However, one sample (Golden shower) showed extremely high antioxidant activity (41.49\%).

Estimation of antioxidant activity by FRAP: The FRAP assay is high-throughput, adaptable and can detect antioxidant capacities as low as $0.2 \mathrm{mM} \mathrm{Fe} 2+$ equivalents. The assay measures the antioxidant potential in samples through the reduction of ferric iron $(\mathrm{Fe} 3+)$ to ferrous iron $(\mathrm{Fe} 2+)$ by antioxidants present in the samples. The lowest antioxidant activity was found in Moringa (37.7888\%). Out of five samples, four samples has antioxidant activity in range of $37.7888-54.4855 \%$. one sample (Golden shower) showed extremely high antioxidant activity (142.577\%).

\section{CONCLUSION}

The purpose of this study is to reevaluate the work done by scientists earlier in this field of plant biotechnology and microbiology to broaden the aspect of plant and plant substances as treatments to common infections in the Indian community. However, it could not be proved by this study that as to what extent these plants are useful in providing medical aid, and confirmatory research by professional scientists is still required. Five plant samples were tested for antimicrobial activity, phenolic content, flavonoid content and antioxidant activity.All the five plant samples did not show any antimicrobial activity against bacterial strains.From the study we can conclude that the plant sample adusa has highest phenolic and flavonoid content as compared to other plant samples. This medicinal plant can be proved to be useful for future pharmaceutical purposes. On the other hand, plant sample golden shower showed highest antioxidant activity from both FRAP and DDPH assays. Due to presence of high antioxidant properties, this medicinal plant could be used for making anticancer drugs for future purposes.

\section{REFERENCES}

Alam MM, Siddiqui MB, Hussian W. (1990) Treatment of diabetes throuherbal drugs in rural India. Fitoterpia. Alhakmani, F., Kumar, S., \& Khan, S. A. (2013). Estimation of total phenolic content, in-vitro antioxidant and antiinflammatory activity of flowers of Moringa oleifera. Asian Pacific Journal of Tropical Biomedicine.

Asolkar LV, Kakkar KK, Chakre OJ. (1992). Second supplement to glossary of Indian medicinal plant with active principles. New Delhi: Publication and 
Information Directorate, CSIR.

Balandrin MF, Kjocke AJ, (1985) Wurtele E et al. Natural plant chemicals:sources of industrial and mechanical materials. Science

Benzie, I. F., \&t Strain, J. J. (1996). The ferric reducing ability of plasma (FRAP) as a measure of "antioxidant power": the FRAP assay. Analytical biochemistry.

Calixto, J. B. (2005). Twenty-five years of research on medicinal plants in Latin America. Journal of Ethnopharmacology.

Chen Y, Han T, Qin L, Rui Y, Zheng H. (2003) Effect of total triterpenes from Centella asiatica on the depression behavior and concentration of amino acid in forced swimming mice. Zhong Yao Cai.

Chew, A. L., Jessica, J. J. A., \& Sasidharan, S. (2012). Antioxidant and antibacterial activity of different parts of Leucas aspera. Asian Pacific Journal of Tropical Biomedicine.

Geneva, (1998). Regulatory situation of herbal medicine. World Health Organization.

Ghazanfar SA, Al Sabahi AMA, (1993), Medicinal plants of northern and central Oman. Econ Bot.

Jan Hudzicki, (2009) Kirby-Bauer Disk Diffusion Susceptibility Test Protocol. Journal of Drug Delivery and Therapeutics

Jiao, H., \& Wang, S. Y. (2000). Correlation of antioxidant capacities to oxygen radical scavenging enzyme activities in blackberry. Journal of agricultural and food chemistry,

Kartni T. In: Cracker LE, Simon JE, editors. Herbs, Spices and Medicinal Plants. Arizona, USA: Oryx Press.

Kirtikar, K.R., Basu, B.D. (1995). Indian Medicinal Plants, Allahabad Bishen Singh and Mahendra pal singh,. Krishnaraju AV, Rao TVN, Sundararaju D et al. (2005) Assessment of bioactivity of Indian medicinal plants using Brine shrimp (Artemiasalina) lethality assay. Int J Appl Sci Eng

Lee, J., Chung, H., Chang, P.-S., \&t Lee, J. (2007).
Development of a method predicting the oxidative stability of edible oils using 2,2-diphenyl-1picrylhydrazyl (DPPH). Food Chemistry.

Meena, Harsahay; Pandey, Hemant Kumar; Pandey, Pankaj; Arya, MaheshChand; Ahmed, Zakwan (2012). Evaluation of antioxidant activity of two important memory enhancing medicinal plants Baccopa monnieri and Centella asiatica. Indian Journal of Pharmacology.

Morimoto S, Nonaka G, Chen R. (1988) The potential of aqueous and isolated fraction from leaves of Cassia fistula Linn. as antibacterial agent. Chem Pharmacol Bull.

Moyo B, Masika PJ, Hugo A, Muchenje V. (2011) Nutritional characterization of Moringa (Moringa oleifera Lam.) leaves. Afr J Biotech.

P. Suresh Kumar1*, S. Sucheta1, V.Sudarshana Deepa1, P. Selvamani2 and S. Latha2 (2008)Antioxidant activity in some selected I ndian medicinal plants. Anna University, Tiruchirappalli.

Sarita Manandhar, Shisir Luitel, Raj Kumar Dahal, (2019) "In Vitro Antimicrobial Activity of Some Medicinal Plants against Human Pathogenic Bacteria”, Journal of Tropical Medicine.

Selvamohan T.* and V. Ramadas* S. Shibila Selva Kishore $^{* *}$, (2012) Antimicrobial activity of selected medicinal plants against some selected human pathogenic bacteria, Advances in Applied Science Research.

Singleton, V. L., Orthofer, R., Lamuela-Raventos, R.M. (1999). Analysis of total phenols and other oxidation substrates and antioxidants by means of Folin-Ciocalteu reagent. Methods Enzymology.

U.P. Claeson, T. Malmfors, G. Wikman, J.G. Bruhn. (2000). Adhatoda vasica: a critical review of ethnopharmacological and toxicological data. J. Ethnopharmacology. 